\title{
Surface reconstructions and transport of epitaxial \\ PtLuSb (001) thin films grown by MBE
}

Sahil J. Patel ${ }^{a}$, John A. Logan ${ }^{a}$, Sean D. Harrington ${ }^{a}$, Brian D. Schultz ${ }^{b}$, Chris J. Palmstrøma,

${ }^{a}$ Materials Department, University of California-Santa Barbara, Santa Barbara, 93106-5050,

United States of America

${ }^{b}$ Department of Electrical and Computer Engineering, University of California-Santa Barbara, Santa Barbara, 93106-9560, United States of America

${ }^{*}$ Correspondence to: cpalmstrom@ece.ucsb.edu 


\section{Abstract}

This work presents the surface reconstructions and transport properties of the topological insulator PtLuSb grown on $\mathrm{Al}_{0.1} \mathrm{n}_{0.9} \mathrm{Sb} / \mathrm{GaAs}$ (001). Two stable surface reconstructions, (1x3) and $c(2 \times 2)$, were observed on PtLuSb (001) surfaces. Antimonydimerization was determined to be the nature of the $(1 \times 3)$ surface reconstruction as evidenced by chemical binding energy shifts in the antimony $4 d$ core-level for surface bonding components. The two surface reconstructions were studied as a function of $\mathrm{Sb}_{4}$ overpressure and substrate temperature to create a reconstruction phase diagram. From this reconstruction phase diagram, a growth window from $320^{\circ} \mathrm{C}$ to $380{ }^{\circ} \mathrm{C}$ using an antimony overpressure was identified. Within this window, the highest quality films were grown at a growth temperature of $380^{\circ} \mathrm{C}$. These films exhibited lower $\mathrm{p}$-type carrier concentrations as well as relatively high hole mobilities.

\section{Keywords}

B1: Heusler compounds; B2: Topological insulator compounds; A3: Molecular beam epitaxy; A1: Surface structure; A1: X-ray photoemission spectroscopy; A1: Reflection high energy electron diffraction 


\section{Introduction}

Heusler compounds are ternary intermetallics, which, depending on the chemical species and ordering, can exhibit novel electronic structures ranging from half-metallic ferromagnets[1-3] to semiconductors[3,4] to superconductors[5]. Despite the wide range of properties, Heusler compounds only form in a few related crystal structures, including the $L 2_{1}$, $\mathrm{B} 2, \mathrm{DO}_{3}$, and $\mathrm{C1}_{\mathrm{b}}$. In addition, these materials are closely lattice matched to III-V semiconductors, enabling integration into existing device technologies. Recently, the existence of topological insulators in the family of half-Heusler compounds has been proposed[6-8]. These materials have an insulating bulk band gap combined with conductive surface states where the electron spin is locked to the momentum. Recent experiments have reported the first direct experimental observation of these topologically non-trivial surface states in Heusler compounds in the topological insulator candidate, PtLuSb[9]. Although there has been significant progress with bulk prepared samples[10], including record mobilities for PtLuSb[11], to utilize and modulate these spin-locked surface states, gateable high quality thin film device structures must be fabricated. During thin film growth, crystalline quality, including bulk defects and surface morphology, is determined by the kinetics of the growing surface; therefore, understanding surface bonding is crucial in determining ideal growth conditions. This work presents the stable surface reconstructions of PtLuSb (001) surfaces and demonstrates how they relate to finding conditions for stoichiometric growth.

\section{Experimental Methods}

PtLuSb (001) films were grown by molecular beam epitaxy (MBE) on relaxed $\mathrm{Al}_{0.1} \mathrm{In}_{0.9} \mathrm{Sb}$ films grown on GaAs (001) substrates. MBE has produced high quality half-Heusler films[12-15] as well as offers the ability to control elemental fluxes and abruptly terminate surfaces. Additionally, MBE provides sub-monolayer deposition control over elemental species, allowing the surface reconstructions of PtLuSb (001) films to be evaluated as a function of the 
termination procedure. $\mathrm{Al}_{0.1} \mathrm{In}_{0.9} \mathrm{Sb}$ buffer layers were chosen due to the similarities between the zinc blende and half-Heusler $\left(\mathrm{C}_{\mathrm{b}}\right)$ crystal structures as well as the ability to lattice match the PtLuSb films[16]. Reflection high-energy electron diffraction (RHEED) was used to monitor film growth and paired with in-situ low-energy electron diffraction (LEED) to study the surface reconstructions of grown films. In-situ x-ray photoemission spectroscopy (XPS) was used for surface composition and impurity detection. Ex-situ characterization included x-ray diffraction (XRD), to examine the bulk crystalline structure, and synchrotron XPS to probe the bulk and surface bonding of the films together with LEED to verify the surface reconstruction prior to the synchrotron XPS measurements.

GaAs (001) substrates were used due to the availability of semi-insulating and highly doped wafers. Semi-insulating wafers were used for lateral transport measurements to limit parallel conduction paths while $\mathrm{p}+\mathrm{GaAs}$ (001) wafers were used for photoemission measurements. Growth conditions were identical for each sample regardless of substrate choice. For growth, the GaAs wafers were loaded into a VG V80H III-V MBE system with a base pressure of $<5 \times 10^{-11}$ mbar, where the native oxide was desorbed under $\mathrm{As}_{4}$ overpressure. A 500 $\mathrm{nm}$ buffer layer of GaAs was grown at a substrate temperature of $600{ }^{\circ} \mathrm{C}$ to form a smooth surface and to trap any remaining surface impurities after desorption of the native oxide. After the buffer layer growth, samples were annealed under an $\mathrm{As}_{4}$ overpressure at $600{ }^{\circ} \mathrm{C}$ to form a well ordered (2x4)- $\beta 2$ arsenic-terminated surface. Following the anneal, the substrate was cooled to $520^{\circ} \mathrm{C}$, at which point arsenic was shuttered. After a 10 minute anneal while residual $\mathrm{As}_{4}$ was pumped out of the chamber, the substrate was further cooled to $380{ }^{\circ} \mathrm{C}$. This cooling procedure retained the arsenic-stabilized $(2 \times 4)-\beta 2$ reconstruction at $380{ }^{\circ} \mathrm{C}$. To nucleate $\mathrm{Al}_{0.1} \mathrm{In}_{0.9} \mathrm{Sb}$, the antimony shutter was opened for a 10 second presoak of the GaAs surface followed by codeposition of $\mathrm{Al}_{0.1} \mathrm{In}_{0.9} \mathrm{Sb}$. Typical group- $\mathrm{V}$ to group-III beam flux ratios were between 1.2 and 1.3 using a valved-cracker antimony source to supply an $\mathrm{Sb}_{2}$ molecular beam. 
Due to the extremely high mismatch of $14.1 \%$ between $\mathrm{Al}_{0.1} \mathrm{In}_{0.9} \mathrm{Sb}$ and $\mathrm{GaAs}$, the film immediately relaxes to form a three-dimensional structure, as observed by RHEED. Following nucleation, the substrate temperature was raised to $420{ }^{\circ} \mathrm{C}$ for continued growth. The higher growth temperature limited the sticking of excess antimony and improved adatom diffusivity. The RHEED pattern became streaky after approximately $15 \mathrm{~nm}$ of growth, indicating the coalescence and formation of a continuous, two-dimensional $\mathrm{Al}_{0.1} \ln _{0.9} \mathrm{Sb}$ surface. The $\mathrm{Al}_{0.1} \mathrm{In}_{0.9} \mathrm{Sb}$ buffer was grown to either a thickness of $19.4 \mathrm{~nm}$ for lateral transport measurements or $200 \mathrm{~nm}$ for XRD and photoemission studies[17]. After growth, the $\mathrm{Al}_{0.1} \mathrm{In}_{0.9} \mathrm{Sb}$ buffers were cooled under an $\mathrm{Sb}_{2}$ overpressure and terminated with a mixed $(1 \times 3) / \mathrm{c}(4 \times 4)$ reconstruction before being transferred in-situ under ultra high vacuum conditions $\left(<5 \times 10^{-10} \mathrm{mbar}\right)$ to a metals MBE chamber (a modified Gen-II EMOF growth chamber with a base pressure $<1 \times 10^{-10}$ mbar) for PtLuSb growth.

For PtLuSb growth, lutetium was evaporated from a high temperature effusion cell using a tantalum crucible, $\mathrm{Sb}_{4}$ was evaporated from a conventional effusion cell using a pyrolytic-BN crucible, and platinum was evaporated from an e-beam evaporator. Source material consisted of elemental platinum (Alfa Aesar Premion 99.99+\% metals basis), lutetium (Ames Laboratory 99.99\% total basis), and antimony (United Mineral Corporation (UMC) $99.99999 \%$ metals basis). For lutetium and antimony, atomic fluxes were measured in-situ using a beam flux gauge with the collector connected to an electrometer for high precision current measurements. For platinum, atomic fluxes were monitored in-situ by a quartz-crystal microbalance located next to the sample. All in-situ flux measurements were correlated to actual atomic fluxes using Rutherford backscattering spectrometry to measure the atomic density of elemental films grown on silicon substrates at room temperature for set periods of time. These correlations were then used prior to each growth in order to set cell temperatures and e-beam emission currents to obtain the desired atomic flux ratios.

To promote the formation of PtLuSb, a shuttered growth technique was employed 
following the $C 1_{b}$ PtLuSb crystal structure: the $z=0$ plane of PtLuSb consists of 2 lutetium and 2 antimony atoms per unit cell and the $z=1 / 4$ plane consists of 2 atoms of platinum. Sequential deposition of $6.74 \times 10^{14}$ atoms $/ \mathrm{cm}^{2}$ of lutetium, followed by $6.74 \times 10^{14}$ atoms $/ \mathrm{cm}^{2}$ of platinum, and finally $6.74 \times 10^{14}$ atoms $/ \mathrm{cm}^{2}$ of antimony (one formula unit of PtLuSb) was repeated four times to form an 8 monolayer template at $\sim 225^{\circ} \mathrm{C}$. This epitaxial template was then used to grow thicker films of PtLuSb by codeposition of platinum, lutetium, and antimony at $320-$ $380^{\circ} \mathrm{C}$. Pt:Lu:Sb flux ratios were set to 1:1:1.3. No evidence of excess antimony incorporation in the films at these growth conditions was observed in RHEED or XRD. This allowed for an antimony overpressure to be maintained during growth, similar to the growth of III-Sb semiconductors[18]. Typical growth rates were around $5 \AA /$ min.

Films were capped with a protective $2 \mathrm{~nm}$ amorphous silicon, $5 \mathrm{~nm} \mathrm{SiO}$, or $100 \mathrm{~nm}$ antimony layer for ex-situ XRD, magneto-transport, or synchrotron XPS measurements, respectively, to prevent PtLuSb oxidation while in atmosphere. Carrier concentration and mobility was studied using by Hall measurements in the Van der Pauw geometry using indium contacts. To further understand the surface chemistry of the different surface reconstructions, synchrotron XPS measurements were taken as a function of incident photon energy at the i4 beamline at MAX-lab in Lund, Sweden. Synchrotron XPS measurement samples also included an additional 5 monolayer thick epitaxial GdSb diffusion barrier[19] between the $\mathrm{Al}_{0.1} \operatorname{In}_{0.9} \mathrm{Sb}$ buffer layer and the PtLuSb film to minimize interfacial reactions during the thermal desorption of the sacrificial antimony cap. Complete thermal desorption of the antimony cap was verified at MAX-lab by LEED and core-level XPS. A (1x3) surface reconstruction, identical to that seen in the growth reconstruction study, was observed following de-cap. XPS spectra were fitted using a convolution of asymmetric Gaussian and Lorentzian line shapes. The full-width at half maximum for each component was held constant at $0.57 \mathrm{eV}$ for $120 \mathrm{eV}$ photon energies and $0.61 \mathrm{eV}$ for $90 \mathrm{eV}$ photon energies. Energy splitting between antimony $4 \mathrm{~d}^{5 / 2}$ and antimony $4 \mathrm{~d}^{3 / 2}$ was constrained to $1.25 \mathrm{eV}$ for all fits. 


\section{Results and Discussion}

\subsection{Structural analysis of PtLuSb (001) thin films}

Beginning with XRD measurements, Figure 1 shows the $\theta-2 \theta$ diffraction pattern for PtLuSb grown on a $200 \mathrm{~nm} \mathrm{Al} \mathrm{l}_{0.1} \mathrm{In}_{0.9} \mathrm{Sb}$ buffer on GaAs (001). The epitaxial relationship for the grown structures was PtLuSb (001) // $\mathrm{Al}_{0.1} \mathrm{In}_{0.9} \mathrm{Sb}$ (001) // GaAs (001): PtLuSb [110] // $\mathrm{Al}_{0.1} \mathrm{In}_{0.9} \mathrm{Sb}$ [110] // GaAs [110]. The absence of non-(00I) reflections confirms the growth of an epitaxial film with a (001) orientation. In addition, the absence of (00I) reflections, where I is odd, is consistent with the half-Heusler crystal structure. (002) and (004) reflections are also observed for the GaAs substrate and the $\mathrm{Al}_{0.1} \mathrm{In}_{0.9} \mathrm{Sb}$ buffer layer. The out-of-plane lattice parameter of the PtLuSb film is measured to be $6.45 \AA$, aligned with the $A \mathrm{l}_{0.1} \mathrm{In}_{0.9} \mathrm{Sb}$, and in good agreement with measurements of bulk crystals[10]. Further, we note the present of finite thickness fringes around both the (002) and the (004) peaks, suggesting the growth of abrupt, high quality interfaces. The film thickness calculated from the fringe spacing is $7.0 \pm 0.1 \mathrm{~nm}$, consistent with the expected growth rate as determined by beam flux calibrations.

\subsection{Surface reconstructions of PtLuSb (001) thin films}

Surfaces were characterized by RHEED and LEED as a function of substrate temperature and antimony overpressure. After post-growth annealing at various antimony overpressures and substrate temperatures, three unique surface phases were observed for PtLuSb (001): (1x3), $c(2 \times 2)$, and antimony capping. RHEED and LEED images of both the (1x3) and $c(2 \times 2)$ reconstructed surfaces show streaky patterns consistent with smooth high quality growth (Figure 2A). By varying both antimony overpressure and substrate temperature, a reconstruction phase diagram was obtained, shown in Figure 2B. (1x3) reconstructions are observed at low antimony overpressures or high substrate temperatures while $c(2 \times 2)$ reconstructions are observed for higher antimony overpressures or lower substrate 
temperatures. The stability of the $c(2 \times 2)$ reconstruction at lower substrate temperatures and higher antimony overpressures than the $(1 \times 3)$ reconstruction indicates that the $c(2 \times 2)$ surface reconstruction contains more antimony as compared to the $(1 \times 3)$ reconstruction. To retain $(1 \times 3)$ reconstructions at room temperature, the substrate temperature was held at approximately 360 ${ }^{\circ} \mathrm{C}$ while the antimony cell was shuttered, and then the sample was cooled to room temperature with no impinging antimony flux. $c(2 \times 2)$ reconstructions were retained at room temperature by cooling to $330{ }^{\circ} \mathrm{C}$ under an antimony overpressure of $8.75 \times 10^{14}$ atoms $/ \mathrm{cm}^{2} \mathrm{~min}$ until a $\mathrm{c}(2 \times 2)$ reconstruction was obtained, and then shuttering antimony and cooling the substrate to room temperature. Further decreasing substrate temperature at constant antimony overpressure eventually results in the formation of elemental antimony on the surface.

\subsection{X-ray photoemission spectroscopy of PtLuSb (001) thin films}

The observation of surface reconstructions that change as a function of antimony overpressure or substrate temperature suggests a change in surface antimony composition for each reconstruction, similar to reconstruction changes observed in III-V semiconductors, like GaAs[20]. Figure 3 shows angle-integrated XPS spectra of the antimony $4 \mathrm{~d}$ core level at incident photon energies of $120 \mathrm{eV}$ (less surface sensitive) and $90 \mathrm{eV}$ (more surface sensitive) for (1x3) reconstructed PtLuSb (001). Two components, S1 and S2, are observed at lower binding energy $\left(\Delta \mathrm{BE}_{\mathrm{S} 1}=-0.46 \pm 0.03 \mathrm{eV}, \Delta \mathrm{BE}_{\mathrm{S} 2}=-0.92 \pm 0.03 \mathrm{eV}\right)$ than the bulk component (bulk antimony $4 d^{5 / 2}=32.21 \pm 0.02 \mathrm{eV}$ ). The peak ratio of the components $\mathrm{S} 1$ and $\mathrm{S} 2\left(\mathrm{~A}^{\mathrm{S} 1} / \mathrm{A}^{\mathrm{S} 2}\right)$ remains nearly constant at 1.1 for both photon energies. However, the total component to bulk ratio $\left(\mathrm{A}^{\mathrm{S} 1+\mathrm{S} 2} / \mathrm{A}^{\text {bulk }}\right)$ at $h v=90 \mathrm{eV}$ is 2.1 as compared to 1.4 at $h v=120 \mathrm{eV}$. Since the $\mathrm{hv}=90 \mathrm{eV}$ measurement is more surface sensitive, the larger total component to bulk ratio confirms that $\mathrm{S} 1$ and S2 arise from bonding at the surface. The S1 component, with a $-0.46 \mathrm{eV}$ shift from the bulk component, is analogous to surface components observed in III-V semiconductors like GaAs (001) and GaSb (001) where a similarly shifted components have been attributed to group-V 
dimerization at the surface[21,22]. The S2 component, which is shifted $-0.92 \mathrm{eV}$ from the bulk component, represents a more negative bonding environment for antimony, and could be attributed to broken antimony dimers leading to unpaired electrons at step edges or other surface defects. Notably, both antimony core-level shifts towards lower binding energies provide compelling evidence to indicate that antimony-antimony dimers are present in the $(1 \times 3)$ surface reconstruction. Antimony de-capping limitations prevented similar analysis with the $c(2 \times 2)$ surface reconstruction, although the reconstruction phase diagram suggests that dimers or an even further antimony saturated surface should be present.

\subsection{Electrical transport of PtLuSb (001) thin films}

The reconstruction phase diagram presented in Figure 2B highlights a growth window of substrate temperatures between $320-410{ }^{\circ} \mathrm{C}$ where an antimony flux of $8.75 \times 10^{14}$ atoms $/ \mathrm{cm}^{2} \mathrm{~min}$ results in a stable antimony-terminated surface without the formation of an elemental antimony capping layer or the appearance of additional features in the RHEED/XRD patterns. This verifies the existence of a growth window where antimony overpressures can be used while still retaining approximately $1: 1: 1 \mathrm{Pt}: \mathrm{Lu}: \mathrm{Sb}$ stoichiometry in the film. Within this growth window, substrate temperature was found to play a large role in electronic film quality, as measured by room temperature carrier concentration and mobility (Figure 4). A clear trend showing decreasing carrier concentration as well as increasing mobility is observed as growth temperature is increased. The purity of the source materials, the ultra high vacuum conditions and the lack of detection of oxygen and carbon or other impurities on the film surface by in-situ XPS suggests that unintentional impurities are unlikely to be the dominant cause of these behaviors. However, both behaviors may be attributed to a reduction of point defects, such as antisites or interstitials, which can introduce carriers and defects that serve as scattering centers that reduce mobility. Furthermore, the observed trends in carrier concentration and mobility point towards growth at a higher substrate temperature, which would increase adatom mobility 
resulting in improved crystallinity as well as reduce the incorporation of excess nonstoichiometric antimony. Unfortunately, above growth temperatures of $380^{\circ} \mathrm{C}$, the PtLuSb halfHeusler phase is not stable on $\mathrm{Al}_{x} \mathrm{In}_{1-x} \mathrm{Sb}$, and significant film-substrate reactions begin to occur. While these reactions provide an upper limit for growth temperature in the $\mathrm{PtLuSb} / \mathrm{Al} \mathrm{I}_{\mathrm{x}} \mathrm{n}_{1-\mathrm{x}} \mathrm{Sb}$ system, it is possible that another substrate could be used that is less reactive with PtLuSb, enabling growth at higher substrates temperatures, potentially resulting in higher quality films. Other studies have used MgO (001) substrates with metallic buffers, such a Ta (001), for higher temperature growths[23], but these layers are conductive, making it difficult to study the electronic structure of the film by lateral transport measurements and limiting access to the helical surface states. Furthermore, the higher symmetry of these metal layers allows for the formation of additional rotational and antiphase defects within the Heusler compound which is undesirable.

\section{Conclusions}

This work demonstrates the optimization of the growth of epitaxial PtLuSb (001) thin films on $\mathrm{Al}_{0.1} \mathrm{In}_{0.9} \mathrm{Sb}(001) / \mathrm{GaAs}(001)$ heterostructures. The observation of both (1x3) and $c(2 \times 2)$ surface reconstructions under an antimony overpressure indicated that these surface reconstructions results from differences in the surface bonding of antimony. XPS analysis suggests some degree of dimerization exists between the surface antimony atoms as evidenced by the observation of energy shifts towards lower binding energy in antimony $4 \mathrm{~d}$ surface components. The stability of the surface reconstructions as a function of substrate temperature and antimony overpressure led to the identification of a growth window were an antimony overpressure could be utilized. For Pt:Lu:Sb atomic flux ratios of 1:1:1.3, a substrate temperature of $320-380^{\circ} \mathrm{C}$ lead to stoichiometric films with the best electrical properties obtained near $380^{\circ} \mathrm{C}$. Further increased growth temperatures lead to film-substrate reactions between the PtLuSb and the $A I_{x} I_{1-x} S b$ buffers used in this study, but may result in further 
improvements in film quality if a more stable epitaxial template can be identified. The use of surface reconstructions to determine a growth window is applicable to the epitaxial growth of other half-Heusler compounds comprised of group-V elements and should enhance their ability to be integrated with various III-V heterostructures.

\section{Acknowledgements}

The authors would like to acknowledge the support of the beamline staff and scientists at the i4 beamline in MAX-LAB. Beamline measurements were partially supported by the IMI Program of the National Science Foundation under Award No. DMR 08-43934. X-ray diffraction measurements were taken at a shared MRSEC funded facility under award number NSF-DMR-1121053. Funding for this work was provided by the Office of Naval Research under award number N00014-11-1-0728 as well as the Army Research Office under award number W911NF-12-1-0459.

\section{References}

[1] S. Picozzi, A. Continenza, A.J. Freeman, Co2MnX (X-Si, Ge, Sn) Heusler compounds: An ab initio study of their structural, electronic, and magnetic properties at zero and elevated pressure, Phys. Rev. B. 66 (2002) 094421. doi:10.1103/PhysRevB.66.094421.

[2] R.A. de Groot, F.M. Mueller, P.G. van Engen, K.H.J. Buschow, New Class of Materials: Half-Metallic Ferromagnets, Phys. Rev. Lett. 50 (1983) 2024-2027. doi:10.1103/PhysRevLett.50.2024.

[3] J. Tobola, J. Pierre, S. Kaprzyk, R. V Skolozdra, M.A. Kouacou, Crossover from semiconductor to magnetic metal in semi-Heusler phases as a function of valence electron concentration, J. Phys. Condens. Matter. 10 (1998) 1013-1032. http://iopscience.iop.org/0953-8984/10/5/011 (accessed August 20, 2014).

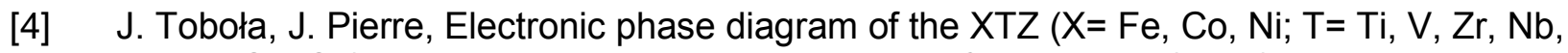
$\mathrm{Mn} ; \mathrm{Z}=\mathrm{Sn}$, Sb) semi-Heusler compounds, J. Alloys Compd. 296 (2000) 243-252. http://www.sciencedirect.com/science/article/pii/S0925838899005496 (accessed August 20, 2014). 
[5] J. Winterlik, G. Fecher, A. Thomas, C. Felser, Superconductivity in palladium-based Heusler compounds, Phys. Rev. B. 79 (2009) 064508.

doi:10.1103/PhysRevB.79.064508.

[6] W. Al-Sawai, H. Lin, R. Markiewicz, L.A. Wray, Y. Xia, S.-Y. Xu, et al., Topological electronic structure in half-Heusler topological insulators, Phys. Rev. B. 82 (2010) 125208. doi:10.1103/PhysRevB.82.125208.

[7] H. Lin, L.A. Wray, Y. Xia, S. Xu, S. Jia, R. Cava, et al., Half-Heusler ternary compounds as new multifunctional experimental platforms for topological quantum phenomena, Nat. Mater. 9 (2010) 546-549. doi:10.1038/nmat2771.

[8] S. Chadov, X. Qi, J. Kübler, G. Fecher, C. Felser, S.-C. Zhang, Tunable multifunctional topological insulators in ternary Heusler compounds, Nat. Mater. 9 (2010) 541-545. doi:10.1038/nmat2770.

[9] J.A. Logan, S.J. Patel, S.D. Harrington, C.M. Polley, B.D. Schultz, B. Thiagarajan, et al., Observation of a topologically non-trivial surface state in half-Heusler PtLuSb (001) thin films, arXiv:1511.04778. (2015).

[10] C. Shekhar, S. Ouardi, G.H. Fecher, A. Kumar Nayak, C. Felser, E. Ikenaga, Electronic structure and linear magnetoresistance of the gapless topological insulator PtLuSb, Appl. Phys. Lett. 100 (2012) 252109. doi:10.1063/1.4730387.

[11] Z. Hou, Y. Wang, G. Xu, X. Zhang, E. Liu, W. Wang, et al., Transition from semiconducting to metallic-like conducting and weak antilocalization effect in single crystals of LuPtSb, Appl. Phys. Lett. 106 (2015) 102102. doi:10.1063/1.4914545.

[12] J.K. Kawasaki, T. Neulinger, R. Timm, M. Hjort, A. a. Zakharov, A. Mikkelsen, et al., Epitaxial growth and surface studies of the Half Heusler compound NiTiSn (001), J. Vac. Sci. Technol. B. 31 (2013) 04D106. doi:10.1116/1.4807715.

[13] P. Bach, A.S. Bader, C. Rüster, C. Gould, C.R. Becker, G. Schmidt, et al., Molecularbeam epitaxy of the half-Heusler alloy NiMnSb on (In, Ga) As/lnP (001), Appl. Phys. Lett. 83 (2003) 521-523. doi:10.1063/1.1594286.

[14] W.H. Wang, M. Przybylski, W. Kuch, L.I. Chelaru, J. Wang, Y.F. Lu, et al., Magnetic properties and spin polarization of Co2MnSi Heusler alloy thin films epitaxially grown on GaAs(001), Phys. Rev. B. 71 (2005) 144416. doi:10.1103/PhysRevB.71.144416.

[15] R. Stephan, F. Dulot, a. Mehdaoui, D. Berling, P. Wetzel, Molecular-beam epitaxy of Heusler alloy thin films epitaxially grown on Si(001), J. Magn. Magn. Mater. 320 (2008) 1043-1049. doi:10.1016/j.jmmm.2007.10.009. 
[16] C.J. Palmstrøm, Epitaxy of dissimilar materials, Annu. Rev. Mater. Sci. 25 (1995) 389415. http://www.annualreviews.org/doi/pdf/10.1146/annurev.ms.25.080195.002133 (accessed January 23, 2014).

[17] S.J. Patel, J.K. Kawasaki, J. Logan, B.D. Schultz, J. Adell, B. Thiagarajan, et al., Surface and electronic structure of epitaxial PtLuSb (001) thin films, Appl. Phys. Lett. 104 (2014) 201603. doi:10.1063/1.4879475.

[18] W.K. Liu, M.B. Santos, Surface reconstructions of InSb (001) during molecular beam epitaxy, Surf. Sci. 319 (1994) 172-183. doi:10.1016/0039-6028(94)90580-0.

[19] B.D. Schultz, H.H. Farrell, M.M.R. Evans, K. Lüdge, C.J. Palmstrøm, ErAs interlayers for limiting interfacial reactions in Fe/GaAs(100) heterostructures, J. Vac. Sci. Technol. B. 20 (2002) 1600-1608. doi:10.1116/1.1491994.

[20] A. Ohtake, Surface reconstructions on GaAs(001), Surf. Sci. Rep. 63 (2008) 295-327. doi:10.1016/j.surfrep.2008.03.001.

[21] I.M. Vitomitrov, A. Raisanen, A.C. Finnefrock, R.E. Viturro, L.J. Brillson, P.D. Kirchner, et al., Geometric ordering, surface chemistry, band bending, and work function at decapped GaAs(100) surfaces, Phys. Rev. B. 46 (1992) 13293.

[22] M.T. Sieger, T. Miller, T.-C. Chiang, Reflection high-energy electron diffraction and photoemission study of GaSb(100) reconstructions, Phys. Rev. B. 52 (1995) 8256-8265. doi:10.1103/PhysRevB.52.8256.

[23] R. Shan, S. Ouardi, G.H. Fecher, L. Gao, A. Kellock, K.P. Roche, et al., Electronic and crystalline structures of zero band-gap LuPdBi thin films grown epitaxially on $\mathrm{MgO}(100)$, Appl. Phys. Lett. 102 (2013) 172401. doi:10.1063/1.4802795.

\section{Figures}

Figure 1. $\theta-2 \theta$ XRD scan showing the (002) and (004) reflections of the GaAs substrate, $\mathrm{Al}_{0.1} \mathrm{In}_{0.9} \mathrm{Sb}$ buffer, and PtLuSb film. (A) Survey scan along (00I) direction. (B) Zoom in of the (004) reflection. The pattern confirms the growth of an epitaxial, (001)-oriented film. Finite thickness fringes correspond to a PtLuSb film thickness of $7.0 \pm 0.1 \mathrm{~nm}$.

Figure 2. (A) RHEED and LEED images of $(1 \times 3)$ and $c(2 \times 2)$ reconstructed surfaces. (B) Reconstruction phase diagram for PtLuSb (001) as a function of antimony overpressure and substrate temperature. The presence of $c(2 \times 2)$ reconstructions at lower temperatures and higher antimony overpressures suggests that it is more antimony-rich than the $(1 \times 3)$ surface reconstruction. 
Figure 3. XPS measurements of the antimony $4 d$ core level as a function of incident photon energy. At (A) $120 \mathrm{eV}$ photon energy, three components are observed: bulk, S1, and S2. The more surface sensitive (B) $90 \mathrm{eV}$ photon energy scan shows an increase in peak area of components S1 and S2 as compared to the bulk component, indicating the surface nature of these components.

Figure 4. Room temperature Hall measurements in the Van der Pauw geometry showing trends of decreasing carrier concentration and increasing mobility with increasing growth temperature. This behavior is attributed the decrease in point defect density and/or an increase in crystal quality at higher growth temperatures. 

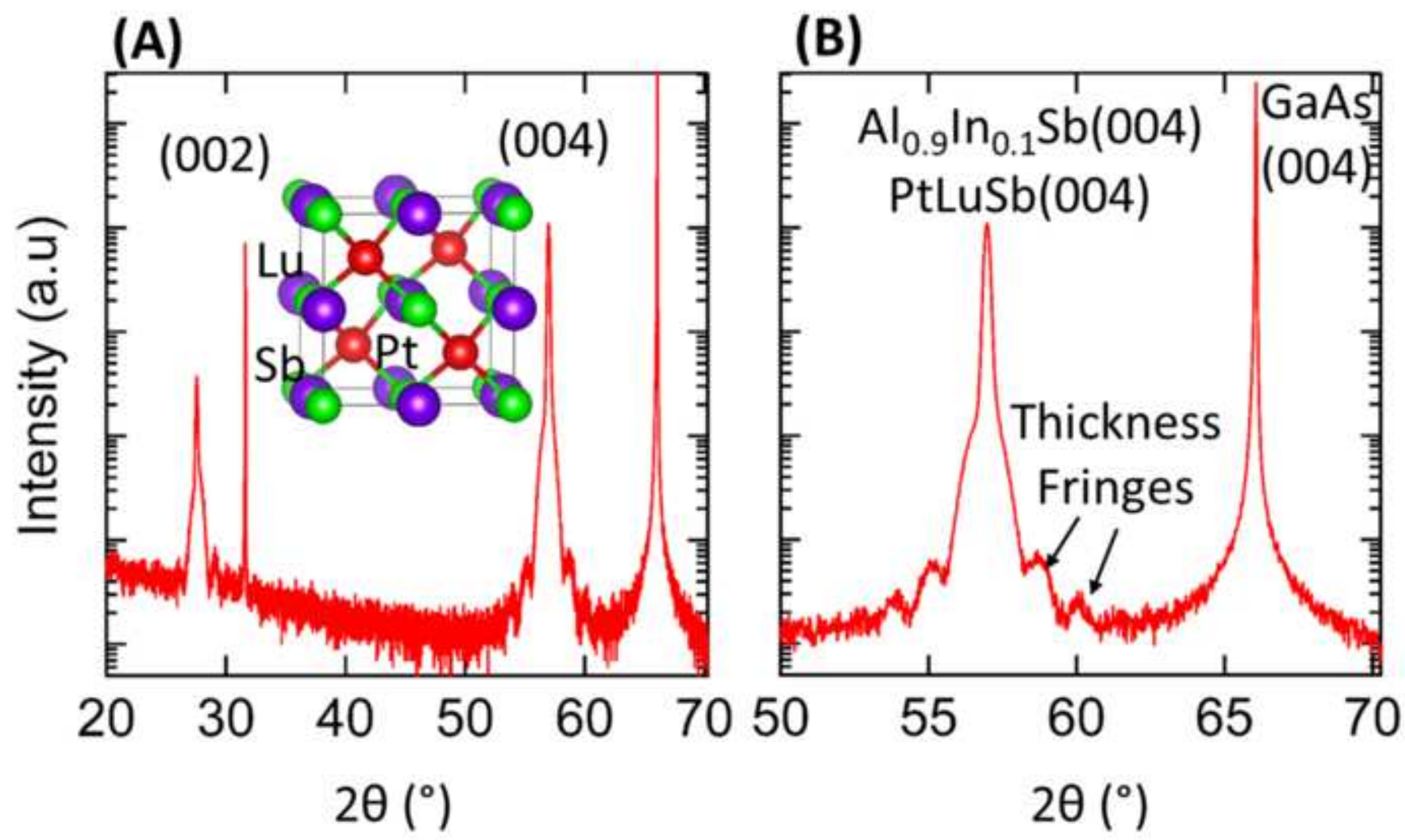

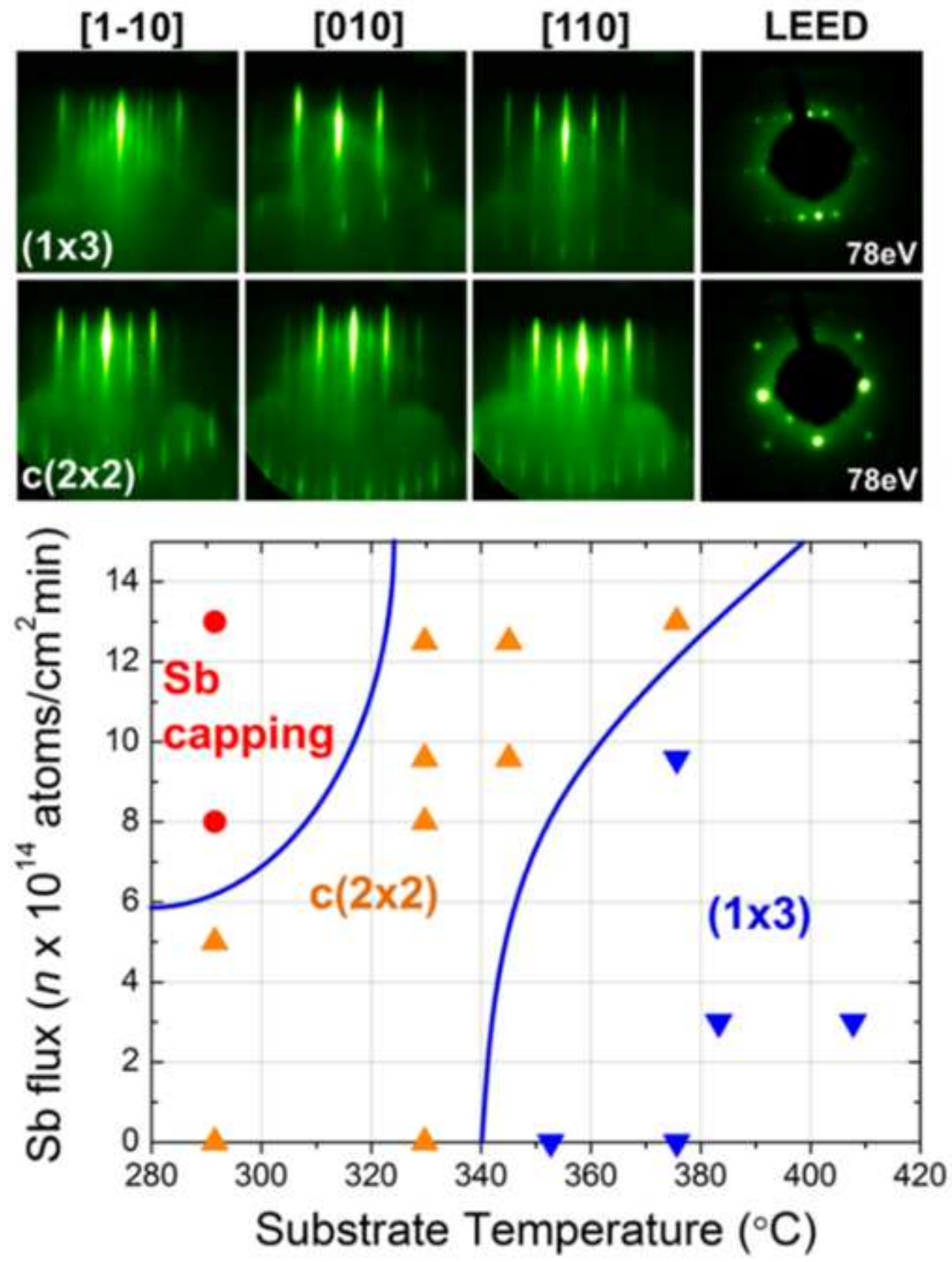


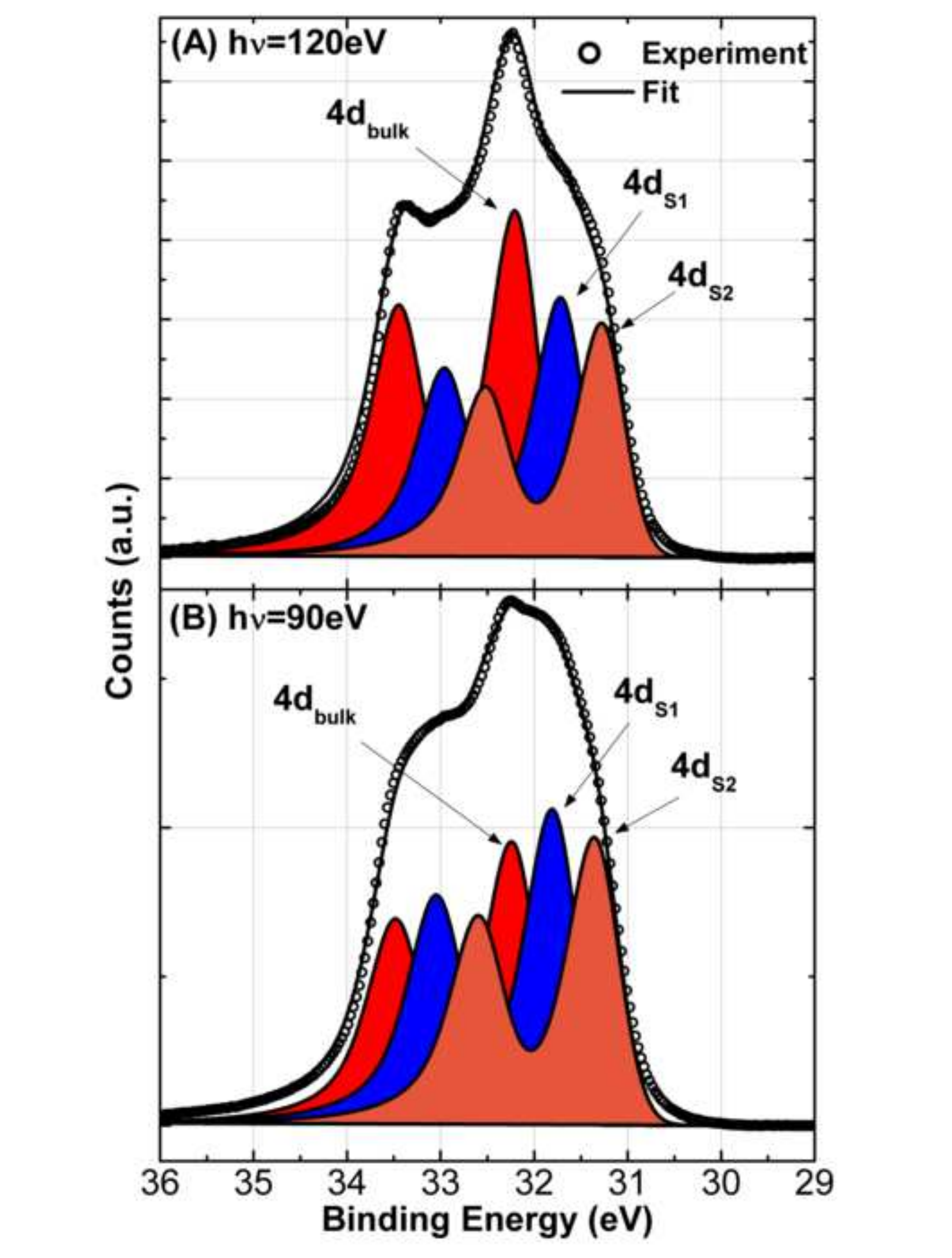

Figure 3

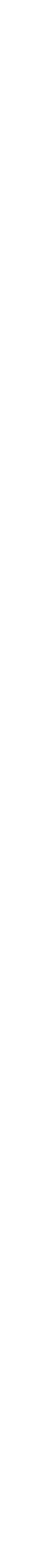
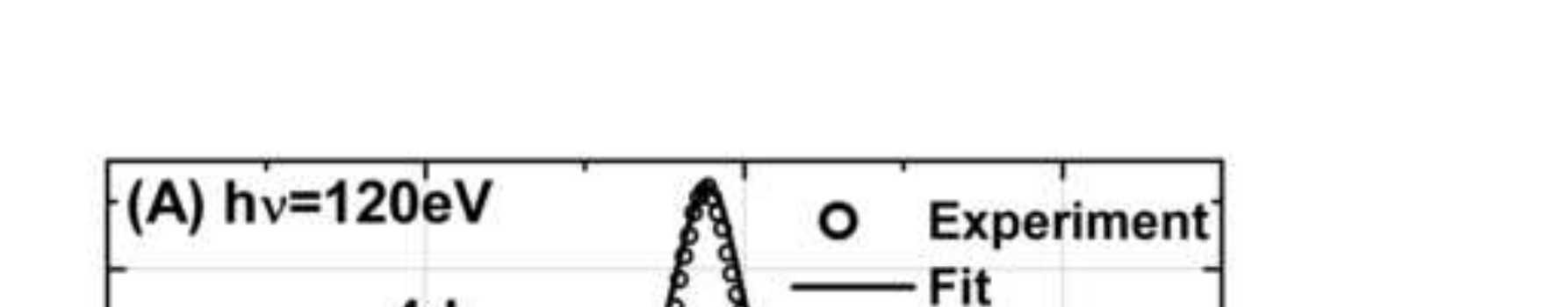

.

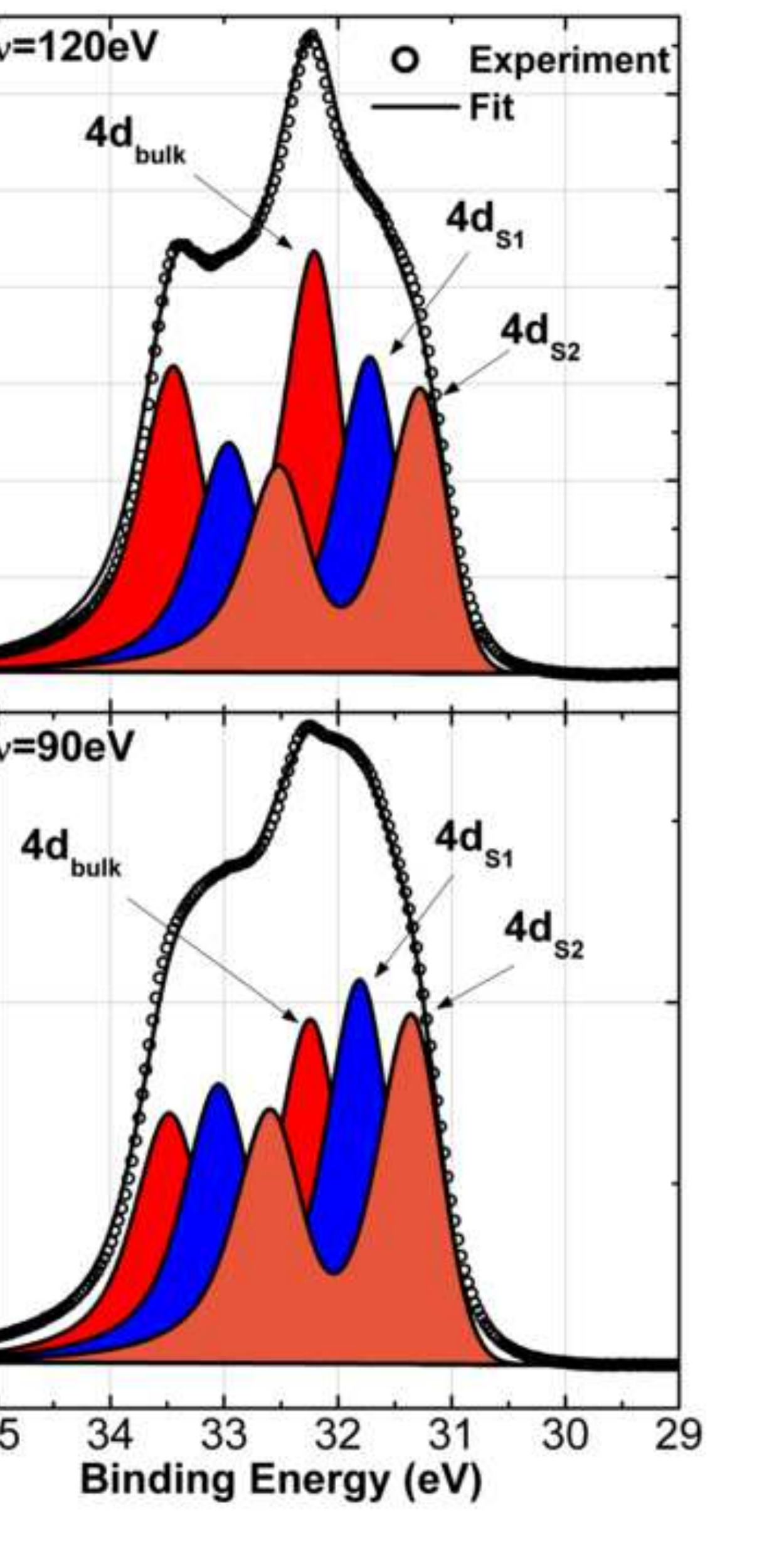


